\title{
Solar System Object Image Search: A precovery search engine
}

\author{
Stephen D. J. Gwyn, Norman Hill and JJ Kavelaars \\ Canadian Astronomy Data Centre, \\ National Research Council \\ 5071 West Saanich Rd, Victoria BC, V9E 2E7, Canada
}

\begin{abstract}
While regular astronomical image archive searches can find images at a fixed location, they cannot find images of moving targets such as asteroids or comets. The Solar System Object Image Search (SSOIS) at the Canadian Astronomy Data Centre allows users to search for images of moving objects, allowing precoveries. SSOIS accepts as input either an object designation, a list of observations, a set of orbital elements, or a user-generated ephemeris for an object. It then searches for observations of that object over a range of dates. The user is then presented with a list of images containing that object from a variety of archives. Initially created to search the CFHT MegaCam archive, SSOIS has been extended to other telescopes including Gemini, Subaru/SuprimeCam, WISE, HST, the SDSS, AAT, the ING telescopes, the ESO telescopes, and the NOAO telescopes (KPNO/CTIO/WIYN), for a total of 24.5 million images. As the PanSTARRS and Hyper Suprime-Cam archives become available, they will be incorporated as well. The SSOIS tool is located on the web at http://www.cadc-ccda.hia-iha.nrc-cnrc.gc.ca/en/ssois/.
\end{abstract}

Keywords. astronomical data bases: miscellaneous, astrometry, minor planets, asteroids, comets: general, celestial mechanics

\section{Introduction}

In many fields of astronomy, the use of archival data is steadily increasing. When stellar or extra-galactic researchers find a new, interesting or anomalous object, the first reaction is to check the archives for images of the object at other wavelengths or higher resolutions. More than $2 / 3 \mathrm{rds}$ of HST papers are at least partially archival in nature. However, while regular archive searches allow users to find images at particular RA and Dec, they cannot find images of moving targets.

The Solar System Object Image Search (SSOIS) at the Canadian Astronomy Data Centre (CADC) meets this need by allowing users to search for images of moving objects. The user enters either a set of observations, an object name, orbital elements or a full ephemeris. SSOIS generates an ephemeris and returns a list of all matching images.

Archival images are potentially quite useful for solar system astronomers. The observed arc of a newly-discovered object can be extended if there pre-existing images can be found of the object. For example, Alexandersen et al. (2012) used SSOIS to refine the orbit of Jovian irregulars. Additionally, some solar system objects change over time; archival images allow studies of the variability of asteroids over longer timescales. For example, Hui and Jewitt (2015) and Hsieh et al. (2015), used SSOIS to find prediscovery observations of active asteroid 313P/(2014 S4) Gibbs to examine the change in activity. Parker et al. (2011) used SSOIS to find additional images of binary TNOs. 


\section{Image collection}

The SSOIS database contains images from multiple telescope archives. While there are similar services that allow users to search for images from a single archive (e.g. the Moving Object Search Tool at IRSA, the Infrared Science Archive, which allows searches on WISE, Spitzer and other IRSA image holdings), the goal of SSOIS is to provide users with a single location to search for all available images, from as many telescopes and instruments as possible. At present, the following telescopes and instruments are searchable via SSOIS:

- CFHT (MegaCam, CFH12K, WIRCam)

- Gemini (GMOS, NIRI)

- HST (WFPC2, ACS, WFC3)

- $\operatorname{INT}(\mathrm{WFC})$

- WHT (AUXCAM, INGRID, LIRIS, Prime)

- JKT (JAG-CCD)

- ESO-LaSilla 2.2m (WFI)

- ESO-NTT (EFOSC, EMMI, SOFI, SUSI, SUSI2)

- ESO-VISTA (VIRCAM)

- ESO-VLT (FORS1, FORS2, HAWKI, ISAAC, NAOS-CONICA, VIMOS)

- ESO-VST (OMEGACAM)

- Subaru (SuprimeCam)

- SDSS

- WISE

- $\operatorname{AAT}(\mathrm{WFI})$

- ALMA

- NEAT-GEODSS

- CTIO-4m (DECam, Mosaic2, NEWFIRM)

- KPNO-0.9m (Mosaic1)

- KPNO-4m (Mosaic1, NEWFIRM)

- WIYN (ODI, MiniMo)

Currently there 24.5 million images listed in the SSOIS archive. In the near future, the Pan-STARRS and Subaru Hyper-SuprimeCam archives will be added to this list.

Different mechanisms are used to retrieve metadata from the different archives. The details of this scraping procedure are discussed in Gwyn (2015)

Metadata for each image is saved:

- The location (RA, Dec and field of view)

- The exposure midpoint in MJD

- Filter

- Exposure time

- The image target name

- The telescope and instrument name

Only the location (RA, Dec and field of view) and the exposure midpoint are required for a search. In programmatic mode, it is possible to query by telescope/instrument. The other parameters are provided as information to the user, but can not be used as constraints at this point in time.

Coverage in the SSOIS database is illustrated by Fig. 1. The coverage is dominated by WISE which covers the whole sky, and the SDSS. The usefulness of a survey instrument is sometimes measured by its étendue, $A \Omega$, its aperture times its field of view. For archival purposes however the usefullness of an instrument is better measured by $A \Omega t$ where $t$ is the number of nights the instrument has been used. 


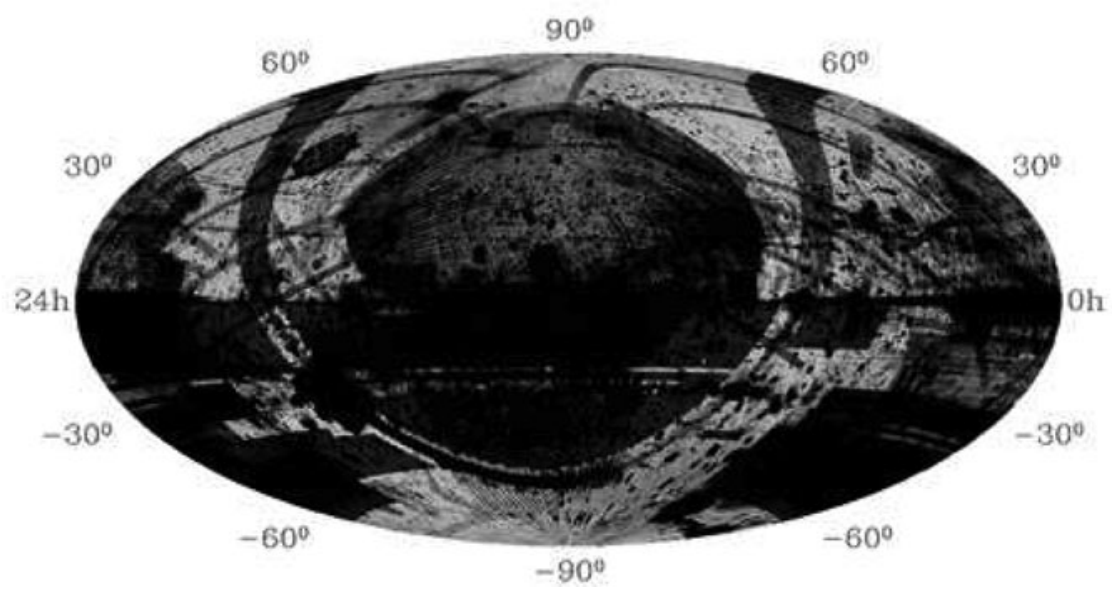

Figure 1. Image coverage in the SSOIS database. The darkness of the greyscale indicates the number of images covering a patch of sky.

\section{Queries}

The SSOIS is available via the CADC website. $\dagger$. The ephemeris to be searched can be entered by one of four methods:

- A user can enter the name, number or provisional designation of an object or objects. SSOIS parses the name against a database of orbital elements regularly updated from the Minor Planet Center (MPC). The ephemeris is computed with orbfit $\ddagger$. Alternatively the user can request that the ephemeris be computed either by the MPC 9 or the Lowell Observatory ephemeris generator $\|$.

- A user can also enter a series of observations in MPC format. SSOIS then fits an orbit to these observations and produces an ephemeris. The ephemeris is either generated using the New Object Ephemeridest† generator from the MPC or using the fit_radec software of Bernstein \& Khushalani (2000).

- A user can enter the orbital elements directly. In this case orbfit will be used to compute the ephemeris.

- Finally, if users mistrust any of the above methods of generating the ephemeris (for example for a newly discovered NEO) they can enter the ephemeris explicitly as a series of times and positions.

The search can be restricted to a short length of time (to speed queries) or can be extended over the full date range of the SSOIS image collection, currently 25 years.

Whichever method is used to generate the ephemeris, SSOIS matches it against the list of images. A temporary table describing the ephemeris is created in the SSOIS database, and joined against the table of archival image. The key to increasing the query speed is to build an integer bounding box around each image in RA, Dec and MJD, and build an index on those three parameters. The ephemeris is also refactored with integer bounding boxes. In the database, a join on the integer values is much faster than a join on floating

\footnotetext{
$\dagger$ http://www.cadc-ccda.hia-iha.nrc-cnrc.gc.ca/en/ssois $\ddagger$ http://adams.dm.unipi.it/ orbmaint/orbfit/

I http://www.cfa.harvard.edu/iau/MPEph/MPEph.html || http://asteroid.lowell.edu/cgi-bin/asteph

$\dagger \dagger$ http://www.cfa.harvard.edu/iau/MPEph/NewObjEphems.html
} 
point numbers. Any matches between the ephemeris and the image list at the integer level are then re-examined at full precision.

The user is presented with a list of images containing the object. Where possible, SSOIS provides direct links to the image in its original archive. In other cases, a link to the relevant search page is provided.

Although originally designed to be used in a web-browser, it is possible to query SSOIS programmatically. A user can add a format=tsv parameter to the URL of the query to get a image listing in tab-separated value format. In programmatic mode, users can also specify that only images from single telescope and instrument be returned, by specifying a telinst parameter (e.g.: telinst=CFHT/MegaCam).

\section{References}

Alexandersen, M., Gladman, B., Veillet, C., Jacobson, R., Brozović, M. \& Rousselot, P., 2012 AJ 144,21

Bernstein, G. \& Khushalani, B., 2000 AJ 120, 3323

Gwyn, S. D. J., 2015 Astronomical Data Analysis Software E3 Systems, p. 413

Hsieh, H. H. et al., 2015 ApJL 800, L16

Hui, M.-T. \& Jewitt, D., 2015, ApJ 149, 134

Parker, A. H., Kavelaars, J. J., Petit, J.-M., Jones, L., Gladman, B., \& Parker, J., 2011, ApJ 743,1 